\title{
UNIQUENESS OF THE CAUCHY PROBLEM FOR LINEAR PARTIAL DIFFERENTIAL EQUATIONS WITH VARIABLE COEFFICIENTS
}

\author{
BY \\ E. C. ZACHMANOGLOU
}

1. Introduction. We consider linear partial differential operators of order $m$ in $n$-dimensional space,

$$
P(x, D)=\sum_{|\alpha| \leqq m} a^{\alpha}(x) D^{\alpha},
$$

where $x=\left(x_{1}, \ldots, x_{n}\right)$ is a point in $R_{n}, \alpha=\left(\alpha_{1}, \ldots, \alpha_{n}\right)$ is an $n$-tuple of integers $\geqq 0$ with $|\alpha|=\sum_{j=1}^{n} \alpha_{j}$ and $D^{\alpha}=D_{1}^{\alpha_{1}} \cdots D_{n}^{\alpha_{n}}$ with $D_{j}=\partial / \partial x_{j}$. The principal part $P_{m}(x, D)$ is the homogeneous part of order $m$,

$$
P_{m}(x, D)=\sum_{|\alpha|=m} a^{\alpha}(x) D^{\alpha}
$$

A surface $\Phi(x)=\Phi\left(x^{0}\right)$, where $\Phi \in C^{1}$ and $\operatorname{grad} \Phi\left(x^{0}\right) \neq 0$, is called characteristic at $x^{0}$ with respect to $P(x, D)$ if

$$
P_{m}(x, \operatorname{grad} \Phi(x))=0 \quad \text { at } x=x^{0} .
$$

If, in addition, one can find a function $\Psi$ such that $P_{m}(x, \operatorname{grad}(\Phi+\varepsilon \Psi))$ is not $O\left(\varepsilon^{2}\right)$ at $x^{0}$ as $\varepsilon \rightarrow 0$, the surface is called simply characteristic at $x^{0}$.

The Cauchy-Kovalevsky theorem asserts that locally, in a neighborhood of a point $x^{0}$ in $R_{n}$, there exists a unique analytic solution of the Cauchy problem for the equation $P(x, D) u=f$, provided that the coefficients of $P(x, D)$, the right-hand side $f$, the initial surface passing through $x^{0}$ and the initial data given on this surface are all analytic in a neighborhood of $x^{0}$ and provided furthermore that the initial surface is not characteristic with respect to $P(x, D)$ at $x^{0}$. The uniqueness assertion of this theorem, with which we are concerned here, was shown by Hörmander [1, Theorem 5.3.1] to be valid even when distribution solutions are allowed.

The condition that the initial surface is not characteristic at $x^{0}$ implies that the surface is not characteristic in some neighborhood of $x^{0}$. On the other hand, if the initial surface is simply characteristic at all of its points in some neighborhood of $x^{0}$, then nonuniqueness of the Cauchy problem holds: there is a smaller neighborhood of $x^{0}$ and a classical solution $u$ of $P(x, D) u=0$ which is defined in this neighborhood, vanishes on one side of the initial surface and is such that $x^{0}$ belongs to the support of $u$.

Received by the editors December 14, 1967. 
In this paper we are interested in the case in which the initial surface is simply characteristic at $x^{0}$ without being characteristic at all of its points in some neighborhood of $x^{0}$. Then, under certain conditions, nonuniqueness of the Cauchy problem holds (see Malgrange [2], Trèves [3, Theorem 6.10] and Zachmanoglou [4]; the last reference deals with partial differential equations with variable coefficients and contains the results of the first two), while under other conditions, uniqueness holds. The first set of conditions for uniqueness were obtained by Hörmander [1, Theorem 5.3.2], who considered equations with variable coefficients. For equations with constant coefficients, more general conditions were obtained by Trèves [3, Theorem 6.9] and Zachmanoglou [5]. The main difficulty in extending the uniqueness theorems in [3] and [5] to equations with variable coefficients lies in the fact that the conditions of these theorems when written for variable coefficients are not invariant under transformations of coordinates. In this paper we formulate and prove a uniqueness theorem with conditions which are invariant under transformations of coordinates. These conditions involve the tangency at $x^{0}$ of the initial surface (which is assumed to be simply characteristic at $x^{0}$ ) with the associated bicharacteristic curve and bicharacteristic strip passing through $x^{0}$.

The uniqueness theorem in this paper contains and extends the results of Hörmander and Trèves. The main idea in the proof is the same as the one used by these authors and is based on a fundamental lemma due to Hörmander [1, Lemma 5.3.2]. The proof involves the construction of a function having noncharacteristic level surfaces. The function used in this paper is simpler than those used by Hörmander and Trèves. It is essentially the same function that was used in [5] in dealing with equations with constant coefficients.

$\S 2$ contains a short review of the main properties of characteristic surfaces and bicharacteristic curves and strips. $\S 3$ contains the statement of the uniqueness theorem and a discussion and proof of the invariance of its conditions for coordinate transformations. The proof is presented in $\$ 4$.

The author wishes to thank Professor F. Trèves and Professor G. Fichera for their valuable advice.

2. Bicharacteristic curves and strips. We present in this section a short review of the definitions and main properties of bicharacteristic curves and strips without proofs. More details and proofs of the assertions in this section may be found, for example in [1, pp. 29-32] and in [4].

Let $P(x, D)$ be a differential operator with principal part $P_{m}(x, D)$ having real $C^{2}$ coefficients defined in an open set $U \subset R_{n}$. We use the notation

$$
P_{m}^{(k)}(x, \xi)=\frac{\partial}{\partial \xi_{k}} P_{m}(x, \xi), \quad P_{m, k}(x, \xi)=\frac{\partial}{\partial x_{k}} P_{m}(x, \xi)
$$

Let $x^{0} \in U$ and $N^{0} \in R_{n}$ be such that $P_{m}\left(x^{0}, N^{0}\right)=0$ and $P_{m}^{(k)}\left(x^{0}, N^{0}\right) \neq 0$ for some $k$. Then a surface passing though $x^{0}$ with normal $N^{0}$ at $x^{0}$ is simply characteristic at 
$x^{0}$. The bicharacteristic strip through $\left(x^{0}, N^{0}\right)$ is the solution $(x(t), \xi(t))$ of the Hamilton equations,

$$
d x_{k} / d t=P_{m}^{(k)}(x, \xi), \quad d \xi_{k} / d t=-P_{m, k}(x, \xi), \quad k=1, \ldots, n,
$$

satisfying the initial conditions

$$
x(0)=x^{0}, \quad \xi(0)=N^{0} .
$$

The curve described by $x$ is called the bicharacteristic curve through $\left(x^{0}, N^{0}\right)$. Clearly this curve is uniquely defined and has no singularities at least in some neighborhood of $x^{0}$.

The condition that $P_{m}(x, \xi)=0$ when $t=0$ implies that $P_{m}(x, \xi)=0$ along the entire strip. Furthermore $\xi$ is normal to the bicharacteristic curve at each of its points. Thus a bicharacteristic strip is a curve (the bicharacteristic curve), which has a specified tangent plane (with normal $\xi$ ) at each of its points.

Let $\Phi$ be a real-valued function in $C^{2}(U)$ such that $\operatorname{grad} \Phi(x) \neq 0$ in $U$ and suppose that the level surfaces of $\Phi$ are simply characteristic everywhere in $U$. If $\operatorname{grad} \Phi\left(x^{0}\right)=N^{0}$, then $\Phi(x)=\Phi\left(x^{0}\right)$ and $\operatorname{grad} \Phi(x)=\xi$ along the entire bicharacteristic strip through $\left(x^{0}, N^{0}\right)$. In geometric terms this means that the bicharacteristic curve through $\left(x^{0}, \operatorname{grad} \Phi\left(x^{0}\right)\right)$ lies on the characteristic surface $\Phi(x)=\Phi\left(x^{0}\right)$ and that the corresponding strip is tangent to the surface along this curve.

\section{Statement and discussion of the uniqueness theorem.}

THEOREM. Let $P(x, D)$ be a differential operator with analytic coefficients defined in a neighborhood $U$ of a point $x^{0}$ in $R_{n}$ and having real coefficients in the principal part. Let $\Phi$ be a real-valued function in $C^{k}(U)$, where $k$ is an integer $\geqq 2$, such that $\operatorname{grad} \Phi\left(x^{0}\right)=N^{0} \neq 0$ and suppose that the surface

$$
S=\left\{x: x \in U, \Phi(x)=\Phi\left(x^{0}\right)\right\}
$$

is simply characteristic with respect to $P(x, D)$ at $x^{0}$. Let $\mathscr{L}=\{x(t), \xi(t)\}$ be the bicharacteristic strip passing through $\left(x^{0}, N^{0}\right)$ when $t=0$, let $l=\{x(t)\}$ be the corresponding bicharacteristic curve, and suppose that

$$
\begin{aligned}
\left.\left(d^{i} / d t^{i}\right)\left[\Phi(x)-\Phi\left(x^{0}\right)\right]\right|_{t=0} & =0, \text { for } i=0, \ldots, k-1, \\
& \neq 0, \text { for } i=k, k \text { odd }, \\
& >0, \text { for } i=k, k \text { even }, \\
\left.\left(d^{i} / d t^{i}\right)[\operatorname{grad} \Phi(x(t))-\xi(t)]\right|_{t=0} & =0, \text { for } i=0, \ldots,[(k-1) / 2] .
\end{aligned}
$$

Then there is a neighborhood $U^{\prime} \subset U$ of $x^{0}$ such that every distribution $u$ defined in $U$, satisfying the equation $P(x, D) u=0$ and vanishing when $\Phi(x)>\Phi\left(x^{0}\right), x \in U$, must also vanish in $U^{\prime}$.

The proof of this theorem is presented in $\$ 4$. We discuss here the geometric meaning of the conditions of the theorem and prove that these conditions are 
invariant for transformations of coordinates. It should be recalled (see [1, pp. 29-32]) that $P_{m}(x, \operatorname{grad} \Phi)$ is invariant for coordinate transformations. In fact $P_{m}(x, \operatorname{grad}(\Phi+\varepsilon \Psi))$ is an invariant for every $\varepsilon$. It follows that the condition that $S$ is simply characteristic at $x^{0}$ is invariant. The equations of a bicharacteristic strip are also invariant for coordinate transformations. The invariance of conditions (6) and (7) follows from their geometric meaning. Condition (6) means that the bicharacteristic curve is a tangent of order $k-1$, and not of higher order, to the surface $S$ at $x^{0}$ and that, when $k$ is even, in a sufficiently small deleted neighborhood of $x^{0}, l$ lies on the side of $S$ where $\Phi(x)>\Phi\left(x^{0}\right)$. Condition (7) means that the bicharacteristic strip $\mathscr{L}$ is tangent of order [(k-1)/2] to the surface $S$ at $x^{0}$ in the direction of $l$.

An alternative expression of condition (7) is also interesting and it will be useful in the proof of the theorem: Let $\Psi$ be any solution of the characteristic equation $P_{m}(x, \operatorname{grad} \Psi)=0$ which is in $C^{[(k+1) / 2]}(U)$ and such that $\operatorname{grad} \Psi\left(x^{0}\right)=N^{0}$. Then, since $\xi(t)=\operatorname{grad} \Psi(x(t))$ (see $\S 2$ ), condition (7) is equivalent to

$$
\left.\left(d^{i} / d t^{i}\right)[\operatorname{grad} \Phi(x(t))-\operatorname{grad} \Psi(x(t))]\right|_{t=0}=0 \quad \text { for } i=0, \ldots,[(k-1) / 2] .
$$

Geometrically this means that any characteristic surface which is tangent to $S$ at $x^{0}$ is also tangent of order $[(k-1) / 2]$ to $S$ at $x^{0}$ in the direction of the bicharacteristic curve through $\left(x^{0}, N^{0}\right)$.

It should be noted that the assumption that $S$ is simply characteristic at $x^{0}$ and equations (4) and (5) defining $\mathscr{L}$ already imply that

$$
\left.\left(d^{i} / d t^{i}\right)\left[\Phi(x(t))-\Phi\left(x^{0}\right)\right]\right|_{t=0}=0 \text { for } i=0,1,
$$

and

$$
\left.[\operatorname{grad} \Phi(x(t))-\xi(t)]\right|_{t=0}=0 .
$$

It is only necessary to verify that $\left.(d / d t) \Phi(x(t))\right|_{t=0}=0$. For $t=0$ we have

$$
\frac{d \Phi}{d t}=\sum_{k=1}^{n} \frac{\partial \Phi}{\partial x_{k}} \frac{d x_{k}}{d t}=\sum_{k=1}^{n} N_{k}^{0} P_{m}^{(k)}\left(x^{0}, N^{0}\right)=m P_{m}\left(x^{0}, N^{0}\right)=0,
$$

where we also used Euler's identity for homogeneous polynomials. $d \Phi / d t=0$ when $t=0$ means that $l$ is tangent to $S$ at $x^{0}$. This of course also follows from the fact that $l$ must lie on all the characteristic surfaces which are tangent to $S$ at $x^{0}$.

When $k=2$, it follows from the above observation that condition (7) and the first two parts of condition (6) are already implied by the assumption that the surface $S$ is simply characteristic at $x^{0}$. Therefore the only other assumption on $S$ is that

$$
\left.\frac{d^{2} \Phi(x(t))}{d t^{2}}\right|_{t=0}>0 .
$$

The value of the left-hand side of (9) can be computed using equations (4) and (5) 
and Euler's identity (see [1, p. 127], or [4]). We have

$$
\left.\frac{d^{2} \Phi}{d t^{2}}\right|_{t=0}=\left.\sum_{i=1}^{n} P_{m}^{(i)}(x, \operatorname{grad} \Phi(x)) \frac{\partial}{\partial x_{i}} P_{m}(x, \operatorname{grad} \Phi(x))\right|_{x=x^{0}}
$$

Since $P_{m}(x, \operatorname{grad}(\Phi+\varepsilon \Psi))$ is an invariant for every $\varepsilon$, the coefficient

$$
\sum_{i=1}^{n} P_{m}^{(i)}(x, \operatorname{grad} \Phi) \frac{\partial \Psi}{\partial x_{i}}
$$

of $\varepsilon$ is also invariant. If $\Psi=P_{m}(x, \operatorname{grad} \Phi)$ this coefficient is equal to the expression on the right-hand side of (10) and this gives another proof of its invariance.

When $k=4$ the only nontrivial part of condition (7) is

$$
\left.(d / d t)[\operatorname{grad} \Phi(x(t))-\xi(t)]\right|_{t=0}=0 .
$$

Again, using equations (4) and (5) we obtain

$$
\left.\left.(d / d t)[\operatorname{grad} \Phi(x(t))-\xi(t)]\right|_{t=0}=\operatorname{grad} P_{m}(x, \operatorname{grad} \Phi(x))\right)\left.\right|_{x=x^{0}} .
$$

It follows that condition (11) is equivalent to the condition that $P_{m}(x, \operatorname{grad} \Phi(x))$ vanishes to the second order at $x^{0}$. This latter condition is obviously invariant for coordinate transformations.

When $k=2$, the theorem coincides with Hörmander's Theorem 5.3.2 in [1]. For differential equations with constant coefficients the theorem coincides with Trèves' Theorem 6.9 in [3].

Before closing this section we remark that the function $\Phi$ in the theorem may be replaced by any other function $\Phi^{\prime}$ provided that $\Phi$ and $\Phi^{\prime}$ define the same surface through $x^{0}$ and the same "side". Specifically let $\Phi$ and $\Phi^{\prime}$ be any two $C^{k}$ functions with nonvanishing gradient in $U$ and such that the sets $\left\{x: x \in U, \Phi(x)<\Phi\left(x^{0}\right)\right\}$ and $\left\{x: x \in U, \Phi^{\prime}(x)<\Phi^{\prime}\left(x^{0}\right)\right\}$ are identical. Then it is clear from the geometric meaning of the conditions of the theorem that if $\Phi$ satisfies these conditions, $\Phi^{\prime}$ also does. An analytical verification of this assertion is indicated in [4].

4. Proof of the theorem. The proof is based on the following lemma due to Hörmander [1, Lemma 5.3.2].

LEMMA. In an open set $\Omega \subset R_{n}$ let $P(x, D)$ have analytic coefficients, and assume that the coefficient of $D_{n}^{m}$ never vanishes in $\Omega$. If $u$ is a distribution in $\Omega$ satisfying the equation $P(x, D) u=0$ in $\Omega_{c}=\left\{x: x \in \Omega, x_{n}<c\right\}$ for some $c$ and if $\Omega_{c} \cap \operatorname{supp} u$ is relatively compact in $\Omega$, then $u=0$ in $\Omega_{c}$.

We first outline the proof of the theorem. Clearly we can choose the coordinates in such a way that $x^{0}=0$ and $N^{0}$ is in the direction of the vector $(0, \ldots, 0,-1)$. Using the hypothesis and the fact that we can choose $U$ as small as we need, we will construct in a neighborhood of $\bar{U}$ a real-valued analytic function of the form

$$
F(x)=f\left(x^{\prime}\right)-x_{n}, \quad f(0)=0, \quad x^{\prime}=\left(x_{1}, \ldots, x_{n-1}\right)
$$

satisfying the following conditions: 
(13) There is a number $c>0$ such that the set

$$
K=\{x: x \in U, \Phi(x) \leqq \Phi(0), F(x) \geqq-c\}
$$

is a compact subset of $U$.

(14) $P_{m}(x, \operatorname{grad} F(x)) \neq 0$, for $x \in U$.

We then make the analytic change of variables

$$
y_{j}=x_{i}, \quad j=1, \ldots, n-1 ; \quad y_{n}=-F(x) .
$$

The inverse substitution is also analytic. Let $u^{\prime}, \Omega, P^{\prime}$, and $K^{\prime}$ be the images of $u, U, P$ and $K$ respectively. Condition (14) means that the level surfaces $F(x)$ $=$ constant are not characteristic with respect to $P$ in $U$. Hence their images, which are the hyperplanes $y_{n}=$ constant are not characteristic with respect to $P^{\prime}$ in $\Omega$. Furthermore, since $K$ is a compact subset of $U$, its image $K^{\prime}$ is a compact subset of $\Omega$. Hence the set $\left\{y: y \in \Omega, y_{n} \leqq c\right\} \cap \operatorname{supp} u^{\prime}$ is a compact subset of $\Omega$ since it is contained in $K^{\prime}$. It now follows from the lemma that $u^{\prime}=0$ in $\left\{y: y \in \Omega, y_{n}<c\right\}$, and therefore $u=0$ in $U^{\prime}=\{x: x \in U, F(x)>-c\}$ which is a neighborhood of 0 since $F(0)=0>-c$. In order to complete the proof it remains only to construct a function $F$ possessing the required properties (13) and (14).

We first consider the following special case: in some neighborhood of 0 the function $\Phi(x)$ is of the form

$$
\Phi(x)=\varphi\left(x^{\prime}\right)-x_{n}, \quad x^{\prime}=\left(x_{1}, \ldots, x_{n-1}\right)
$$

with $\varphi(0)=0$ and $\operatorname{grad} \Phi(0)=N^{0}=(0, \ldots, 0,-1)$, and the principal part of the differential operator $P(x, D)$ is of the form

$$
P_{m}(x, D)=(-1)^{m-1} D_{n-1} D_{n}^{m-1}+\cdots,
$$

where the dots stand for terms of order less than $m-1$ with respect to $D_{n}$. The assumption on $P_{m}(x, D)$ implies that in a neighborhood of 0 , the planes $x_{n}=$ constant are simply characteristic with respect to $P(x, D)$ and the corresponding bicharacteristic curves on these planes are parallel to the $x_{n-1}$-axis. In fact (see $\S 3$ ) the bicharacteristic strip $\mathscr{L}$ through $\left(0, N^{0}\right)$ is given by the equations

$$
\begin{aligned}
& x_{1}=\cdots=x_{n-2}=x_{n}=0, \quad x_{n-1}=t ; \\
& \xi_{1}=\cdots=\xi_{n-1}=0, \quad \xi_{n}=-1 .
\end{aligned}
$$

Thus, conditions (6) and (7) become in this case

$$
\begin{aligned}
{\left[\left(\partial^{i} / \partial x_{n-1}^{i}\right) \Phi(x)\right]_{x=0} } & =0, \quad \text { for } i=0,1, \ldots, k-1, \\
& \neq 0, \quad \text { for } i=k, k \text { odd }, \\
& >0, \quad \text { for } i=k, k \text { even, }
\end{aligned}
$$

$\left.\left(\partial^{i} / \partial x_{n-1}^{i}\right)\left[\operatorname{grad} \Phi(x)-N^{0}\right]\right|_{x=0}=0$, for $i=0,1, \ldots,[(k-1) / 2]$. 
First we obtain an estimate for the function $\varphi\left(x^{\prime}\right)$. Expanding it in a finite Taylor series, we have

$$
\varphi\left(x^{\prime}\right)=Q_{0}\left(x_{n-1}\right)+\sum_{j=1}^{n-2} Q_{j}\left(x_{n-1}\right) x_{j}+Q\left(x^{\prime \prime}, x_{n-1}\right)+o\left(\left|x^{\prime \prime}\right|^{2}+\left|x_{n-1}\right|^{k}\right)
$$

where $x^{\prime \prime}=\left(x_{1}, \ldots, x_{n-2}\right), Q_{0}$ is a polynomial of degree $\leqq k$ in $x_{n-1}$, the $Q_{j}$, $j=1, \ldots, n-2$, are polynomials of degree $\leqq k-1$ in $x_{n-1}$ and $Q\left(x^{\prime \prime}, x_{n-1}\right)$ is a polynomial of degree $\leqq k$ in $\left(x^{\prime \prime}, x_{n-1}\right)$ without terms of degree $\leqq 1$ with respect to $x^{\prime \prime}$. It follows from condition $\left(6^{\prime}\right)$ that

$$
Q_{0}\left(x_{n-1}\right)=c_{0} \sigma^{k} x_{n-1}^{k}, \quad \sigma= \pm 1,
$$

where $c_{0}$ is a positive constant, and from condition $\left(7^{\prime}\right)$ that there are constants $C_{1}, C_{2}$ and $\varepsilon_{1}$, with $\varepsilon_{1} \rightarrow 0$ as $\left|x^{\prime \prime}\right|,\left|x_{n-1}\right| \rightarrow 0$, such that

$$
\varphi\left(x^{\prime \prime}\right) \geqq c_{0} \sigma^{k} x_{n-1}^{k}-C_{1}\left|x_{n-1}\right|^{q}\left|x^{\prime \prime}\right|-C_{2}\left|x^{\prime \prime}\right|^{2}-\varepsilon_{1}\left|x_{n-1}\right|^{k},
$$

where $q=[(k+1) / 2]$.

We take as neighborhood of the origin the cylindrical set,

$$
U=\left\{x:\left(x^{\prime \prime}, x_{n-1}\right) \in U_{0},-z_{0}<x_{n}<z_{0}\right\}
$$

where

and set

$$
U_{0}=\left\{\left(x^{\prime \prime}, x_{n-1}\right):\left|x^{\prime \prime}\right|<x_{0},-t_{1}<x_{n-1}<t_{0}\right\}
$$

$$
F(x)=f\left(x^{\prime}\right)-x_{n} \text { with } f\left(x^{\prime}\right)=\sigma \delta x_{n-1}-\left|x^{\prime \prime}\right|^{2} / \varepsilon .
$$

We will show that the positive constants $x_{0}, t_{0}, t_{1}, z_{0}, \delta$ and $\varepsilon$ can be chosen sufficiently small so that conditions (13) and (14) are satisfied. It should be noted that when $k$ is even we are free to choose $\sigma$ as either +1 or -1 whereas when $k$ is odd, $\sigma$ is given as either +1 or -1 .

In order to satisfy condition (13) it is sufficient to choose the constants such that the boundaries $\partial S$ and $\partial S^{\prime}$ of the surfaces $S=\{x: x \in U, \Phi(x)=0\}$ and $S^{\prime}=$ $\{x: x \in U, F(x)=-c\}$ lie on the lateral surface $\left\{x: x^{\prime} \in \partial U_{0},-z_{0}<x_{n}<z_{0}\right\}$ of the boundary of $U$ and such that $\partial S^{\prime}$ lies "below" $\partial S$. Since the first requirement can be satisfied simply by choosing $z_{0}$ large in comparison to $x_{0}, t_{0}$ and $t_{1}$, we concentrate on the second, more difficult one. We must choose the constants such that on $\partial S^{\prime}$ the condition $\Phi(x)>0$ holds.

On $\partial S^{\prime}$ we have

$$
\Phi(x)=\Phi(x)-[F(x)+c]=\varphi\left(x^{\prime}\right)-\sigma \delta x_{n-1}+\left|x^{\prime \prime}\right|^{2} / \varepsilon-c
$$

and using the estimate (17),

$$
\Phi(x) \geqq c_{0} \sigma^{k} x_{n-1}^{k}-C_{1}\left|x_{n-1}\right|^{q}\left|x^{\prime \prime}\right|-C_{2}\left|x^{\prime \prime}\right|^{2}-\varepsilon_{1}\left|x_{n-1}\right|^{k}-\sigma \delta x_{n-1}+\left|x^{\prime \prime}\right|^{2} / \varepsilon-c .
$$

In fact, if $\varepsilon$ is sufficiently small, we have the estimate

$$
\Phi(x) \geqq c_{0} \sigma^{k} x_{n-1}^{k}-C_{1}\left|x_{n-1}\right|^{q}\left|x^{\prime \prime}\right|-\varepsilon_{1}\left|x_{n-1}\right|^{k}-\sigma \delta x_{n-1}+\left|x^{\prime \prime}\right|^{2} / 2 \varepsilon-c .
$$


First, we consider $k$ even, in which case $q=k / 2$, and set

$$
\delta=t_{0}^{k-1+(1 / 4)}, \quad x_{0}=t_{0}^{(k / 2)+(1 / 4)}, \quad \varepsilon=t_{0}^{(1 / 2)}, \quad t_{1}=t_{0} .
$$

We state once and for all that all the following estimates are valid provided $t_{0}$ is sufficiently small. On the parts of $\partial S^{\prime}$ on which $\left|x^{\prime \prime}\right| \leqq x_{0}, x_{n-1}= \pm t_{0}$ we have

$$
\begin{aligned}
\Phi(x) & \geqq c_{0} t_{0}^{k}-C_{1} t_{0}^{(k / 2)} x_{0}-\varepsilon_{1} t_{0}^{k}-\delta t_{0}-c \\
& =t_{0}^{k}\left[c_{0}-C_{1} t_{0}^{(1 / 4)}-\varepsilon_{1}-t_{0}^{(1 / 4)}\right]-c>\frac{c_{0}}{2} t_{0}^{k}-c .
\end{aligned}
$$

On the parts of $\partial S^{\prime}$ on which $\left|x^{\prime \prime}\right|=x_{0},-t_{1} \leqq x_{n-1} \leqq t_{0}$ we have

$$
\begin{aligned}
\Phi(x) & \geqq\left(c_{0}-\varepsilon_{1}\right) x_{n-1}^{k}-C_{1}\left|x_{n-1}\right|^{(k / 2)} x_{0}-\delta\left|x_{n-1}\right|+x_{0}^{2} /(2 \varepsilon)-c \\
& \geqq-C_{1} t_{0}^{k+(1 / 4)}-t_{0}^{k+(1 / 4)}+t_{0}^{k} / 2-c>\frac{1}{4} t_{0}^{k}-c .
\end{aligned}
$$

Thus, when $k$ is even, condition (13) is satisfied with $c=\min \left[c_{0}, \frac{1}{2}\right] t_{0}^{k} / 2$.

Next we consider $k$ odd, in which case $q=(k+1) / 2$. We discuss only the case $\sigma=+1$. The proof for $\sigma=-1$ can be obtained from the proof for $\sigma=+1$ by interchanging $t_{0}$ and $t_{1}$ throughout. We set

$$
\delta=t_{0}^{k-1+(1 / 8)}, \quad x_{0}=t_{0}^{(k-1) / 2+(1 / 4)}, \quad \varepsilon=t_{0}^{(1 / 8)}, \quad t_{1}=t_{0}^{1+(1 / 8)} .
$$

On the part of $\partial S^{\prime}$ on which $\left|x^{\prime \prime}\right| \leqq x_{0}, x_{n-1}=t_{0}$ we have (as in the case of $k$ even)

$$
\Phi(x)>c_{0} t_{0}^{k} / 2-c .
$$

On the part of $\partial S^{\prime}$ on which $\left|x^{\prime \prime}\right| \leqq x_{0}, t=-t_{1}$ we have

$$
\begin{aligned}
\Phi(x) & \geqq-\left(c_{0}+\varepsilon_{1}\right) t_{1}^{k}-C_{1} t_{1}^{(k+1) / 2} x_{0}+\delta t_{1}-c \\
& =t_{0}^{k+(1 / 4)}\left[1-\left(c_{0}+\varepsilon_{1}\right) t_{0}^{(k-2) / 8}-C_{1} t_{0}^{(k+1) / 16}\right] \\
& >\frac{1}{2} t_{0}^{k+(1 / 4)}-c .
\end{aligned}
$$

On the part of $\partial S^{\prime}$ on which $\left|x^{\prime \prime}\right|=x_{0},-t_{1} \leqq t \leqq t_{0}$ we have

$$
\begin{gathered}
\Phi(x) \geqq-c_{0} t_{1}^{k}-C_{1} t_{0}^{(k+1) / 2} x_{0}-\varepsilon_{1} t_{0}^{k}-\delta t_{0}+x_{0}^{2} /(2 \varepsilon)-c \\
=\frac{1}{2} t_{0}^{k-(5 / 8)}-c_{0} t_{0}^{k+(k / 8)}-C_{1} t_{0}^{k+(1 / 4)}-\varepsilon_{1} t_{0}^{k} \\
-t_{0}^{k+(1 / 8)}-c>\frac{1}{4} t_{0}^{k-(5 / 8)}-c .
\end{gathered}
$$

Thus, when $k$ is odd, condition (3) is satisfied with $c=\frac{1}{2} t_{0}^{k+(1 / 4)}$.

Next, we verify that with the constants chosen as above the function $F$ satisfies condition (14). We have

$$
\operatorname{grad} F(x)=\left(-2 x^{\prime \prime} / \varepsilon, \sigma \delta,-1\right) .
$$

From the assumption (16) it follows that

$$
P_{m}\left(x ; \xi^{\prime \prime}, \xi_{n-1},-1\right)=\xi_{n-1}+\cdots
$$

where the dots stand for terms of degree $\geqq 2$ with respect to $\left(\xi^{\prime \prime}, \xi_{n-1}\right)$. Hence

$$
P_{m}\left(x ; \xi^{\prime \prime}, \xi_{n-1},-1\right)=\xi_{n-1}\left[1+O\left(\left|\xi^{\prime \prime}\right|+\left|\xi_{n-1}\right|\right)\right]+O\left(\left|\xi^{\prime \prime}\right|^{2}\right),
$$


in a neighborhood of $x=\xi^{\prime \prime}=\xi_{n-1}=0$. Consequently

$$
P_{m}(x, \operatorname{grad} F)=\sigma \delta\left[1+O\left(\left|x^{\prime \prime}\right| / \varepsilon+\delta\right)\right]+O\left(\left|x^{\prime \prime}\right|^{2} / \varepsilon^{2}\right)
$$

in a neighborhood of 0 . For sufficiently small $t_{0}, U$ is contained in this neighborhood. Now, it is easy to verify that there is a positive constant $C_{3}$ such that when $k$ is even, the above choice of constants and $\sigma=1$ yield

$$
P_{m}(x, \operatorname{grad} F) \geqq \frac{1}{2} t_{0}^{k-1+(1 / 4)}-C_{3} t_{0}^{k-1+(1 / 2)}>0
$$

in $U$ for sufficiently small $t_{0}$. When $k$ is odd and $\sigma=+1$,

$$
P_{m}(x, \operatorname{grad} F) \geqq \frac{1}{2} t_{0}^{k-1+(1 / 8)}-C_{3} t_{0}^{k-1+(1 / 4)}>0
$$

in $U$. When $k$ is odd and $\sigma=-1$, we have in a similar way $P_{m}(x, \operatorname{grad} F)<0$ in $U$. The proof of the theorem for the special case considered is complete.

It remains to reduce the general case to the case that we have studied. We do this by means of analytic transformations of coordinates. Let $\Psi(x)$ be a real analytic function defined in a neighborhood of 0 and satisfying the conditions

$$
P_{m}(x, \operatorname{grad} \Psi)=0, \quad \Psi(0)=0, \quad \operatorname{grad} \Psi(0)=N^{0} .
$$

That such a function exists follows from the existence theorem for the initial value problem for the characteristic equation. (See, for example, Theorem 1.8.2 and the remark following it in [1].) Since $N^{0}$ is in the direction of $(0, \ldots, 0,-1)$, the equations

$$
\begin{aligned}
& y_{j}=x_{j}, \quad j=1, \ldots, n-1, \\
& y_{n}=-\Psi(x),
\end{aligned}
$$

define a nonsingular analytic transformation of coordinates in a neighborhood of 0 . The level surfaces $\Psi(x)=$ constant which are simply characteristic with respect to $P(x, D)$ in a neighborhood of $x=0$ are mapped to the hyperplanes $y_{n}=$ constant which therefore are simply characteristic with respect to the image $P^{\prime}(y, D)$ of $P(x, D)$ in a neighborhood of $y=0$. Since $P_{m}^{\prime(j)}(0 ; 0, \ldots, 0,-1) \neq 0$ for some $j$, $j=1, \ldots, n$, and since $P_{m}^{\prime(n)}(0 ; 0, \ldots, 0,-1)=0$ we may assume, renaming the variables if necessary, that $P_{m}^{\prime(n-1)}(0 ; 0, \ldots, 0,-1) \neq 0$.

Now, in a neighborhood of $y=0$, the equations of the bicharacteristic curve with parameter $t=z_{n-1}$, lying on the plane $y_{n}=z_{n}$ and passing through the point $y^{\prime \prime}=z^{\prime \prime}$, $y_{n-1}=0, y_{n}=z_{n}$ when the parameter $t=z_{n-1}=0$, are of the form

$$
\begin{aligned}
y_{j} & =z_{j}+f_{j}\left(z^{\prime \prime}, z_{n-1}, z_{n}\right), \\
y_{n-1} & =f_{n-1}\left(z^{\prime \prime}, z_{n-1}, z_{n}\right), \\
y_{n} & =z_{n} .
\end{aligned}
$$

The functions $f_{j}$ are analytic in a neighborhood of $z=\left(z^{\prime \prime}, z_{n-1}, z_{n}\right)=0$ and $f_{j}\left(z^{\prime \prime}, 0, z_{n}\right)=0, j=1, \ldots, n-1$. Moreover, it follows from Hamilton's equations, that the Jacobian of $y$ with respect to $z$ at the origin is equal to

$$
P_{m}^{\prime(n-1)}(0 ; 0, \ldots, 0,-1) \neq 0 \text {. }
$$


Hence, the analytic mapping $z \rightarrow y$ defined by the above equations, has an analytic inverse $y \rightarrow z$ in a neighborhood of 0 . It is easy to verify that in the $z$-coordinate system the planes $z_{n}=$ const are simply characteristic with respect to the image $P_{m}^{\prime \prime}(z, D)$ of $P_{m}^{\prime}(y, D)$ in a neighborhood of 0 , and the corresponding bicharacteristic curves on these planes are parallel to the $z_{n-1}$ axis. In fact, in view of Hamilton's equations we have

$$
P_{m}^{\prime \prime}(z ; 0, \ldots, 0,-1)=0
$$

and

$$
\begin{aligned}
P_{m}^{\prime \prime(j)}(z ; 0, \ldots, 0,-1) & =0, \quad \text { for } j=1, \ldots, n-2, n, \\
& =1, \quad \text { for } j=n-1,
\end{aligned}
$$

when $z$ is in some neighborhood of 0 and hence, in this neighborhood,

$$
P_{m}^{\prime \prime}(z, D)=(-1)^{m-1} D_{n-1} D_{n}^{m-1}+\cdots
$$

where the dots stand for terms of order less than $m-1$ with respect to $D_{n}$.

In the $z$-coordinate system the image of the function $\Phi(x)$, which for simplicity we will denote by $\Phi(z)$, is such that grad $\Phi(0)$ is nonzero and in the direction of the vector $(0, \ldots, 0,-1)$. Hence, we may solve the equation $\Phi(z)=\Phi(0)$ for $z_{n}$ to obtain $z_{n}=\varphi\left(z^{\prime}\right)$, where $z^{\prime}=\left(z_{1}, \ldots, z_{n-1}\right), \varphi(0)=0, \varphi_{z_{j}}(0)=0, j=1, \ldots, n-1$, and $\varphi \in C^{k}$ in some neighborhood of 0 . If $\Phi^{\prime}(z)=\varphi\left(z^{\prime}\right)-z_{n}$, then the sets $\{z: z \in U, \Phi(z)<\Phi(0)\}$ and $\left\{z: z \in U, \Phi^{\prime}(z)<0\right\}$ are identical provided $U$ is a sufficiently small neighborhood of 0 . According to the remarks at the end of $\S 3$, we may replace the function $\Phi$ with the function $\Phi^{\prime}$. Now, in the $z$-coordinate system all the assumptions of the special case considered above are satisfied. Since the conditions of the theorem are invariant for analytic transformations of coordinates and since the inverse transformations are also analytic, the proof of the theorem is complete.

The work presented in this paper was done under National Science Foundation Grants GP-06208 and GP-07422.

\section{BIBLIOGRAPHY}

1. L. Hörmander, Linear partial differential operators, Academic Press, New York, 1963.

2. B. Malgrange, Sur les ouverts convexes par rapport à un opérateur différentiel, C. R. Acad. Sci. Paris 254 (1962), 614-615.

3. F. Trèves, Linear partial differential equations with constant coefficients, Gordon and Breach, New York, 1966.

4. E. C. Zachmanoglou, Nonuniqueness of the Cauchy problem for linear partial differential equations with variable coefficients, Arch. Rational Mech. Anal. 27 (1968), 373-384.

5. - Uniqueness of the Cauchy problem when the initial surface contains characteristic points, Arch. Rational Mech. Anal. 23 (1966), 317-326.

Purdue University, LAFAYETTE, INDIANA 